\title{
PDL1 restoration reverses T1DM
}

Most immunotherapy approaches to cure type 1 diabetes mellitus (T1DM) tested to date have failed, probably owing to lack of specificity. However, the transplantation of autologous haematopoietic stem and progenitor cells (HSPCs) has shown promise. "This led us to further explore this field and focus our studies on characterizing HSPCs in T1DM and in nonobese diabetic (NOD) mice," explains lead investigator Paolo Fiorina.

Fiorina and colleagues have discovered a defect in the expression of programmed cell death 1 ligand 1 (PDL1) - an immune checkpoint protein that inhibits $\mathrm{T}$ cell activation - in HSPCs, and demonstrate that genetic overexpression or pharmacological restoration of PDL1 is a viable immunotherapy strategy to reverse T1DM.

Using transcriptomic profiling, PDL1 expression was found to be lower in HSPCs isolated from NOD mice than in those from normal mice. Subsequent genome-wide profiling revealed that 14 PDL1-related microRNAs (miRNAs) were differentially expressed in HSPCs obtained

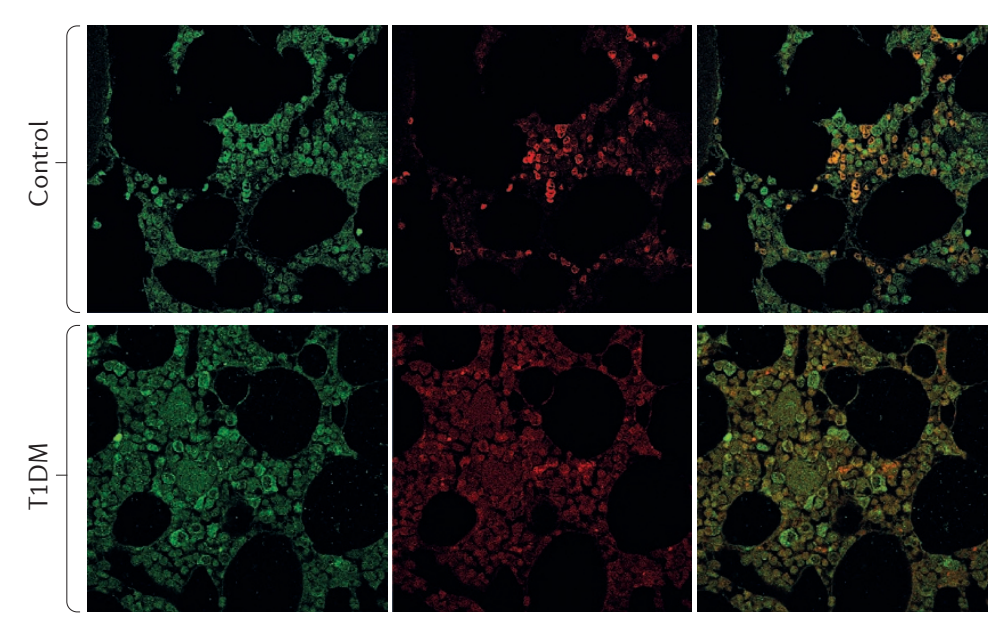

Confocal imaging of bone marrow sections from patients with T1DM and healthy controls showing PDL1 (green) and CD34 (red) staining. Image courtesy of M. Ben Nasr, Harvard Medical School, Boston, USA.

from NOD and normal mice, suggesting that the PDL1 defect was due to an altered miRNA network. Indeed, silencing of one such miRNA restored PDL1 expression in HSPCs.

Next, HSPCs from NOD mice were engineered to overexpress PDL1 (PDL1.Tg). Compared with untransduced HSPCs, PDL1.Tg HSPCs markedly decreased IFN $\gamma$ positive $\mathrm{CD}^{+} \mathrm{T}$ cell numbers when co-cultured with naive $T$ cells in the presence of an islet autoantibody, which was abrogated using an anti-PDL1 antibody. In addition, PDL1.Tg HSPCs induced apoptosis in autoreactive $\mathrm{CD}^{+}$and $\mathrm{CD} 8^{+} \mathrm{T}$ cells. Adoptive transfer of PDL1.Tg HSPCs into newly hyperglycaemic NOD mice reversed hyperglycaemia in $100 \%$ of mice, with long-term reversal observed in $\sim 30 \%$ of mice; none of those that received untransduced HSPCs reverted to normoglycaemia. The pancreata of PDL1.Tg HSPC-treated NOD mice exhibited reduced islet infiltration, preserved insulin staining and an improved insulitis score, compared with untreated mice, and tracking studies suggested that PDL1.Tg HSPCs selectively trafficked to the inflamed pancreas.

As an alternative to gene therapy, HSPCs were also pharmacologically modulated (pHSPCs) using a cocktail of agents that robustly upregulated PDL1 expression. These pHSPCs also decreased the percentage of IFN $\gamma$ positive $\mathrm{CD}^{+} \mathrm{T}$ cells, which was mitigated using an anti-PDL1 antibody. Furthermore, adoptive transfer of pHSPCs reversed diabetes in $40 \%$ of newly hyperglycaemic NOD mice, with similar improvements in pancreatic pathology to those observed in the PDL1.Tg HSPCs model.

Consistent with the preclinical data, fewer PDL $1^{+} \mathrm{CD} 34^{+}$cells were detectable in the peripheral blood and bone marrow of patients with T1DM than in healthy controls. Additional gene expression profiling also validated the presence of an altered miRNA network in these CD $34^{+}$cells. Importantly, pharmacological modulation of CD $34^{+}$ $\left(\mathrm{pCD}^{+} 4^{+}\right.$cells upregulated PDL1 expression, and an anti-PDL1 antibody mitigated $\mathrm{pCD} 34^{+}$-dependent decreases in IFN $\gamma$-producing cells during co-culture with CD34-depleted peripheral blood mononuclear cells.

"Our study will offer a novel therapy for T1DM, although it requires further refinement and optimization to eventually permit its safe translation to the bedside," concludes Fiorina. "Our future studies will focus on understanding the mechanism controlling PDL1 expression in HSPCs, which could also have a role in T1DM pathogenesis".

Conor A. Bradley

ORIGINAL ARTICLE Ben Nasr, M. et al. PD-L1 genetic overexpression or pharmacological restoration in hematopoietic stem and progenitor cells reverses autoimmune diabetes. Sci. Transl. Med. http://dx.doi.org/10.1126/scitranslmed.aam7543 (2017) 